\title{
The q-integers and the Mersenne numbers
}

\section{Amelia Carolina Sparavigna}

Politecnico di Torino

\begin{abstract}
Here we will show that the q-integers, the q-analogue of the integers that we can find in the q-calculus, are forming an additive group having a generalized sum similar to the sum of the Tsallis q-entropies of independent systems. The symmetric form of q-integers will be studied too. We will see that these numbers are linked to the Kaniadakis Ikappa-calculus. In the article, a final discussion will be devoted to the link of the q-integers to the Mersenne numbers. Besides the discussion of the previously mentioned numbers, the general aim of the paper is that of popularizing the existence of the q-calculus.
\end{abstract}

Keywords q-calculus, q-integers, Tsallis q-entropy, Mersenne numbers.

Written in Turin, on May 23, 2018

Submitted SSRN, on May 23, 2018

Introduction Several mathematicians have contributed to a calculus that today is known as the q-calculus [1-6]. As a consequence of the many contributions, we find that it is known as "quantum calculus," or "time-scale calculus", or "calculus of partitions" too [5]. It is also called the "calculus without limits", because it is equivalent to the traditional infinitesimal calculus without the notion of limits. Besides being known with different names, the q-calculus is expressed by means of different notations or, as told in [5], by different "dialects". Here we will use the approach and the notation given in the book by Kac and Cheung [6].

The first aim of the work here proposed is that of showing the following fact. The q-integers, the q-analogue of the integers that we can find in the q-calculus, are forming a group having a generalized sum which is similar to sum of the Tsallis q-entropies of independent systems. After, we will see that the symmetric form of q-integers is linked to the Kaniadakis calculus. We will conclude the discussion considering the Mersenne numbers and their link to the q-integers. Let us stress that, besides the discussion of the previously mentioned numbers, the general aim of the paper is that of popularizing the existence of the q-calculus.

\section{The q-integers}

Let us start defining the q-integers.

In the q-calculus, the q-difference is simply given by:

$$
d_{q} f=f(q x)-f(x)
$$


From this difference, the q-derivative is given as:

$$
\text { (1) } D_{q} f=\frac{f(q x)-f(x)}{q x-x}
$$

The q-derivative reduces to the Newton's derivative in the limit $q \rightarrow 1$. (1) is also known as the Jackson derivative, after Frank Hilton Jackson (1870 - 1960), the English clergyman and mathematician who worked at the beginning of the XXth century on the q-calculus.

Let us consider the function $f(x)=x^{n}$. If we calculate its q-derivative, we obtain:

$$
\text { (2) } D_{q} x^{n}=\frac{(q x)^{n}-x^{n}}{q x-x}=\frac{q^{n}-1}{q-1} x^{n-1}
$$

Comparing the ordinary calculus, which is giving $\left(x^{n}\right)^{\prime}=n x^{n-1}$, to Equation (2), we can define the "q-integer" $[n]$ by:

$$
\text { (3) } \quad[n]=\frac{q^{n}-1}{q-1}=1+q+q^{2}+\ldots+q^{n-1}
$$

Therefore Equation (2) turns out to be:

$$
D_{q} x^{n}=[n] x^{n-1}
$$

As a consequence, the $n$-th q-derivative of $f(x)=x^{n}$, which is obtained by repeating $n$ times the q-derivative, generates the q-factorial:

$$
[n] !=[n][n-1] \ldots[3][2][1]
$$

Form the q-factorials, we can define q-binomial coefficients:

$$
\frac{[n] !}{[m] ![n-m] !}
$$

This means that we can use the usual Taylor formula, replacing the derivatives by the qderivatives and the factorials by q-factorials (in a previous work, we have discussed the qexponential and q-trigonometric functions [7]). Then, in the q-calculus, the q-integer [ $n$ ] acts as the integer in the ordinary calculus. 


\section{The group of q-integers}

We known that the set of integers consisting of the numbers ..., $-4,-3,-2,-1,0,1,2,3,4, \ldots$, having as operation the addition, is a group. Therefore, let us consider the set of q-integers given by (3) and investigate its group. In particular, we have to determine its operation of addition.

Let us remember that a group is a set $A$ having an operation $\cdot$ which is combining the elements of $A$. That is, the operation combines any two elements $a, b$ to form another element of the group denoted $a \cdot b$. To qualify $(A, \bullet)$ as a group, the set and operation must satisfy the following requirements. Closure: For all $a, b$ in $A$, the result of the operation $a \cdot b$ is also in $A$. Associativity: For all $a, b$ and $c$ in $A$, it holds $(a \cdot b) \cdot c=a \cdot(b \bullet c)$. Identity element: An element $e$ exists in $A$, such that for all elements $a$ in $A$, it is $e \cdot a=a \cdot e=a$. Inverse element: For each $a$ in $A$, there exists an element $b$ in $A$ such that $a \cdot b=b \cdot a=e$, where $e$ is the identity (the notation is inherited from the multiplicative operation).

A further requirement is the commutativity: For all $a, b$ in $A, a \cdot b=b \cdot a$. In this case, the group is known as an Abelian group.

Therefore, to qualify a group as an Abelian group, the set and operation must satisfy five requirements which are known as the Abelian group axioms. A group having a noncommutative operation is called a "non-abelian group" or "non-commutative group". For an Abelian group, one may choose to denote the group operation by + and the identity element by 0 (neutral element) and the inverse element as $-a$ (opposite element). In this case, the group is called an additive group.

First, we have to define the operation of addition. It is not the sum that we use for the integers, but it is a generalized sum which obeys the axioms of the group.

Let us start from the q-integer $[m+n]$ :

$$
\begin{gathered}
{[m+n]=\frac{q^{m+n}-1}{q-1}=\frac{1}{q-1}\left(q^{m} q^{n}-1+q^{m}-q^{m}\right)=\frac{1}{q-1}\left(q^{m}\left(q^{n}-1\right)+q^{m}-1\right)} \\
{[m+n]=\frac{1}{q-1}\left(q^{m}\left(q^{n}-1\right)+\left(q^{m}-1\right)+\left(q^{n}-1\right)+\left(1-q^{n}\right)\right)=\frac{1}{q-1}\left(\left(q^{m}-1\right)\left(q^{n}-1\right)+\left(q^{m}-1\right)+\left(q^{n}-1\right)\right)}
\end{gathered}
$$

Therefore, we have:

(4) $[m+n]=[m]+[n]+(q-1)[m][n]$

Then, we can define the generalized "sum" of the group as:

(5) $[m] \oplus[n]=[m]+[n]+(q-1)[m][n]$ 
(for other examples of generalized sums see [8]).

If we use (5) as the sum, we have the closure of it, because the result of the sum is a qinteger. Moreover, this sum is commutative.

The neutral element is:

$$
\text { (6) }[0]=\frac{q^{0}-1}{q-1}=0
$$

Let us determine the opposite element $[o]$, so that:

$$
\begin{aligned}
& {[o] \oplus[n]=0} \\
& 0=[0]=[o] \oplus[n]=[o]+[n]+(q-1)[o][n] \\
& -[n]=[o]+(q-1)[o][n] \\
& \text { (7) }[o]=-\frac{[n]}{1+(q-1)[n]}=-\frac{q^{n}-1}{(q-1) q^{n}}=\frac{q^{-n}-1}{q-1}=[-n]
\end{aligned}
$$

The opposite element of q-integer $[n]$ is the q-integer of $-n$, that is $[-n]$. Let us discuss the associativity of the sum.

It is necessary to have:

$$
[m] \oplus([n] \oplus[l])=([m] \oplus[n]) \oplus[l]
$$

Let us calculate:

$$
\begin{gathered}
{[m] \oplus([n] \oplus[l])=[m] \oplus([n]+[l]+(q-1)[n][l])} \\
{[m] \oplus([n] \oplus[l])=[m]+[n]+[l]+(q-1)[n][l]+(q-1)[m][n]+(q-1)[m][l]+(q-1)^{2}[m][n][l]}
\end{gathered}
$$

And also:

$$
\begin{gathered}
([m] \oplus[n]) \oplus[l]=([m]+[n]+(q-1)[m][n]) \oplus[l] \\
([m] \oplus[n]) \oplus[l]=[m]+[n]+(q-1)[m][n]+[l]+(q-1)[m][l]+(q-1)[n][l]+(q-1)^{2}[m][n][l]
\end{gathered}
$$

It is also easy to see that:

$$
[m] \oplus[n] \oplus[l]=[m+n+l]
$$


As we have shown, the five axioms of an Abelian group are satisfied. In this manner, using the generalized sum given by (5), we have the Abelian group of the q-integers.

\section{The link to Tsallis calculus}

Let us also note that the generalized sum (5) is similar to the sum that we find in the approach to entropy proposed by Constantino Tsallis.

In 1948 [9], Claude Shannon defined the entropy $S$ of a discrete random variable $\equiv$ as the expected value of the information content: $S=\sum_{i} p_{i} I_{i}=-\sum_{i} p_{i} \log _{b} p_{i}$ [10]. In this expression, $l$ is the information content of $\Xi$, the probability of $i$-event is $p_{i}$ and $b$ is the base of the used logarithm. Common values of the base are 2, the Euler's number e, and 10.

Constantino Tsallis generalized the Shannon entropy in the following manner [11]:

$$
S_{q}=\frac{1}{q-1}\left(1-\sum_{i} p_{i}^{q}\right)
$$

Given two independent systems $A$ and $B$, for which the joint probability density satisfies:

$$
p(A, B)=p(A) p(B)
$$

the Tsallis entropy gives:

(8) $S_{q}(A, B)=S_{q}(A)+S_{q}(B)+(1-q) S_{q}(A) S_{q}(B)$

The sum of more than two terms of Tsallis entropies is discussed in [12].

The parameter $(1-q)$, in a certain manner, measures the departure from the ordinary additivity, which is recovered in the limit $q \rightarrow 1$.

Actually the group on which is based the Tsallis entropy, and therefore Equation (8), is known as the "multiplicative group" [7,13,14]. As stressed in [15], the use of a group structure allows to determine a class of generalized entropies. Let us note the group of the q-integers, with addition (5), can be considered a "multiplicative group" too.

Let us stress that we have a link of the multiplicative group to the Tsallis entropy. The group of the $n$-integers had been studied in $[16,17]$ too, but in these articles, a quite different expression for the generalized sum had been proposed. It is given as the "quantum sum" $[x] \oplus[y]=[x]+q^{x}[y] \quad$, where the link to the Tsallis calculus is less evident. 


\section{Symmetric q-numbers}

In the previous discussion we have considered the group of the q-integers as defined by qcalculus. In [6] it is also defined the symmetric q-integer in the following form (here we use a notation different from that given in the Ref.6):

$$
\text { (9) }[n]_{s}=\frac{q^{n}-q^{-n}}{q-q^{-1}}
$$

Repeating the approach previously given, we can determine the group of the symmetric qintegers.

Let us start from the q-integer $[m+n]_{s}$, which is according to (9):

$$
[m+n]_{s}=\frac{q^{m+n}-q^{-(m+n)}}{q-q^{-1}}
$$

and try to find it as a generalized sum of the q-integers $[m]_{s}$ and $[n]_{s}$.

By writing $q=\exp (\log q)$, the q-integer turns out into a hyperbolic sine:

$$
\text { (10) }[n]_{s}=\frac{q^{n}-q^{-n}}{q-q^{-1}}=\frac{e^{n \log q}-e^{-n \log q}}{q-q^{-1}}=2 \frac{\sinh (n \log q)}{\left(q-q^{-1}\right)}
$$

Apart from a numerical factor, this is the form of the generalized numbers proposed by $G$. Kaniadakis in his $\kappa$-calculus [18-22].

From (10), we can write also:

$$
\frac{1}{2}\left(q-q^{-1}\right)[n]_{s}=\sinh (n \log q)
$$

Therefore:

$$
[m+n]_{s}=\frac{q^{m+n}-q^{-(m+n)}}{q-q^{-1}}=2 \frac{\sinh ((m+n) \log q)}{\left(q-q^{-1}\right)}
$$

Using the properties:

$$
\sinh (x+y)=\sinh x \cosh y+\cosh x \sinh x \quad ; \quad \cosh x=\sqrt{1+\sinh ^{2} x}
$$

we obtain: 


$$
\begin{gathered}
{[m+n]_{s}=\frac{2}{\left(q-q^{-1}\right)}[\sinh (m \log q) \cosh (n \log q)+\sinh (n \log q) \cosh (m \log q)]} \\
{[m+n]_{s}=[m]_{s} \cosh (n \log q)+[n]_{s} \cosh (m \log q)} \\
{[m+n]_{s}=[m]_{s} \sqrt{1+\sinh ^{2}(n \log q)}+[n]_{s} \sqrt{1+\sinh ^{2}(m \log q)}}
\end{gathered}
$$

Let us define: $k=\left(q-q^{-1}\right) / 2$ and then: $k[n]_{s}=\sinh (n \log q)$. As a consequence, we have the generalized sum of the symmetric q-integers as:

$$
\text { (11) }[m]_{s} \oplus[n]_{s}=[m]_{S} \sqrt{1+k^{2}[n]_{S}^{2}}+[n]_{S} \sqrt{1+k^{2}[m]_{S}^{2}}
$$

Let us stress that (11) is also the generalized sum proposed by $\mathrm{G}$. Kaniadakis in the framework of a calculus [19-22], the details of which are given in [22].

By means of (11), we can repeat the approach given previously for q-numbers (3) and study of the group of the symmetric q-integers.

\section{The Mersenne numbers}

In the case that $q=2$, we have:

$$
[n]=\frac{2^{n}-1}{2-1}=2^{n}-1
$$

These are the Mersenne Numbers. About these numbers, a large literature exists (see for instance that given in [23]). Among these numbers we find the Mersenne primes.

The numbers are named after Marin Mersenne (1588 - 1648), a French Minim friar, who studied them in the early 17 th century.

Mersenne numbers are written as [23]:

$$
M_{n}=2^{n}-1
$$

Of course, because they are q-integers for $q=2$, we have the generalized sum given in (5):

$$
\text { (5') }[m] \oplus[n]=[m]+[n]+(2-1)[m][n]=[m]+[n]+[m][n]
$$

But we can repeat the calculus as an exercise. 
We can start from the number $M_{m+n}$ and calculate.

$$
\begin{gathered}
M_{m+n}=2^{m+n}-1 \\
M_{m+n}=2^{m+n}-1=2^{m} 2^{n}-1-2^{m}+2^{m}-2^{n}+2^{n}-1+1=2^{m}\left(2^{n}-1\right)-1+2^{m}-2^{n}+1+2^{n}-1 \\
M_{m+n}=\left(2^{m}-1\right)\left(2^{n}-1\right)+2^{m}-1+2^{n}-1
\end{gathered}
$$

Therefore, we can write the following generalized sum:

$$
M_{m+n}=M_{m} \oplus M_{n}=\left(2^{m}-1\right)\left(2^{n}-1\right)+\left(2^{m}-1\right)+\left(2^{n}-1\right)
$$

or:

$$
\text { (12) } \quad M_{m+n}=M_{m} \oplus M_{n}=M_{m}+M_{n}+M_{m} M_{n}
$$

(12) is the same as ( $\left.5^{\prime}\right)$. Let us stress once more that this is a generalized sum that we can find in the case of the multiplicative groups [8].

Using (12), we can imagine for the Mersenne numbers the following recursive relation:

$$
M_{n+1}=M_{n} \oplus M_{1}=M_{n}+M_{1}+M_{n} M_{1}
$$

We can verify as follow:

$$
2^{n+1}-1=\left(2^{n}-1\right)+\left(2^{1}-1\right)+\left(2^{n}-1\right)\left(2^{1}-1\right)=2^{n}+2^{n+1}-2^{n}-2+1=2^{n+1}-1
$$

The sum (12) is associative, so that:

$$
M_{m} \oplus M_{n} \oplus M_{l}=M_{m}+M_{n}+M_{l}+M_{m} M_{n}+M_{n} M_{l}+M_{m} M_{l}+M_{m} M_{n} M_{l}
$$

We cannot have a group of the Mersenne numbers, without considering also the opposites of them, so that:

$$
0=M_{n} \oplus \text { Opposite }\left(M_{n}\right)
$$

Therefore:

$$
\operatorname{Opposite}\left(M_{n}\right)=-\frac{M_{n}}{M_{n}+1}=M_{-n}
$$

Explicitly:

$$
\operatorname{Opposite}\left(2^{n}-1\right)=-\frac{\left(2^{n}-1\right)}{\left(2^{n}-1\right)+1}=\frac{\left(-2^{n}+1\right)}{2^{n}}=2^{-n}-1
$$

These numbers are the Mersenne numbers with a negative exponent. So we have: 


$$
\begin{gathered}
M_{n-n}=M_{n} \oplus M_{-n}=M_{n}+M_{-n}+M_{n} M_{-n} \\
0=2^{0}-1=\left(2^{n}-1\right)+\left(2^{-n}-1\right)+\left(2^{n}-1\right)\left(2^{-n}-1\right)=2^{n}+2^{-n}-2+2^{n} 2^{-n}-2^{-n}-2^{n}+1=0
\end{gathered}
$$

\section{Symmetric Mersenne}

Let us consider the symmetric q-integer in the case of $q=2$.

We can define the symmetric Mersenne in the following manner:

$$
M_{n}^{s}=[n]_{s}=\frac{2^{n}-2^{-n}}{2-2^{-1}}
$$

By writing $2=\exp (\log 2),(13)$ turns out into a hyperbolic sine:

$$
\text { (14) } M_{n}^{s}=\frac{2^{n}-2^{-n}}{2-2^{-1}}=\frac{e^{n \log 2}-e^{-n \log 2}}{2-2^{-1}}=2 \frac{\sinh (n \log 2)}{\left(2-2^{-1}\right)}
$$

Again, as previously told, apart from a numerical factor, this is the form of the generalized numbers proposed by $\mathrm{G}$. Kaniadakis.

Let us define $k=\left(2-2^{-1}\right) / 2 \quad$; we have the generalized sum of the symmetric Mersenne as:

$$
\text { (15) } M_{m}^{s} \oplus M_{n}^{s}=M_{m}^{s} \sqrt{1+k^{2}\left(M_{n}^{s}\right)^{2}}+M_{n}^{s} \sqrt{1+k^{2}\left(M_{m}^{s}\right)^{2}}
$$

Of course, we have again the generalized sum proposed by G. Kaniadakis.

As a conclusion we can note that, by means of the generalized sums, we have found a different approach to the Mersenne numbers too. In my opinion, it is also possible that it was the form of the Mersenne numbers that inspired the Reverend Jackson to modify the usual derivative into the definition (1) of the q-calculus.

\section{References}

1. Ernst, T. (2012). A Comprehensive Treatment of q-Calculus, Springer Science \& Business Media.

2. Annaby, M. H., \& Mansour, Z. S. (2012). q-Fractional Calculus and Equations, Springer.

3. Ernst, T. (2000). The History of q-calculus and a New Method. Department of Mathematics, Uppsala University.

4. Aral, A., Gupta, V., \& Agarwal, R. P. (2013). Applications of q-Calculus in Operator Theory. Springer Science \& Business Media.

5. Ernst, T. (2008). The different tongues of q-calculus. Proceedings of the Estonian Academy of Sciences, 2008, 57, 2, 81-99 DOI: 10.3176/proc.2008.2.03 
6. Kac, V., \& Pokman Cheung (2002). Quantum Calculus, Springer, Berlin.

7. Sparavigna, A. C. (2016). Graphs of q-exponentials and q-trigonometric functions. HAL Id: hal$01377262 \mathrm{https}: / /$ hal.archives-ouvertes.fr/hal-01377262

8. Sparavigna, A. C. (2018). Generalized Sums Based on Transcendental Functions. SSRN Electronic Journal (May 4, 2018). Available at SSRN: https://ssrn.com/abstract=3171628

9. Shannon, C. E. (1948). A Mathematical Theory of Communication. Bell System Technical Journal 2

(3):379-423. DOI: 10.1002/j.1538-7305.1948.tb01338.x

10. Borda, M. (2011). Fundamentals in Information Theory and Coding. Springer. ISBN 978-3-64220346-6.

11. Tsallis, C. (1988). Possible Generalization of Boltzmann-Gibbs Statistics, Journal of Statistical Physics, 52: 479-487. DOI:10.1007/BF01016429

12. Sparavigna, A. C. (2015). On the generalized additivity of Kaniadakis entropy. International Journal of Sciences, 4(02), 44-48. DOI: 10.18483/ijSci.627

13. Sicuro, G., \& Tempesta, P. (2016). Groups, information theory, and Einstein's likelihood principle. Phys. Rev. E 93, 040101(R).

14. Tempesta, P. (2015). Groups, generalized entropies and L-series. Templeton Workshop on Foundations of Complexity, Oct. 2015. http://www.cbpf.br/ complex/Files/talk_tempesta.pdf

15. Curado, E. M., Tempesta, P., \& Tsallis, C. (2016). A new entropy based on a group-theoretical structure. Annals of Physics, 366, 22-31.

16. Nathanson, M. B. (2006). Additive number theory and the ring of quantum integers. In General theory of information transfer and combinatorics (pp. 505-511). Springer Berlin Heidelberg. Available at https://arxiv.org/pdf/math/0204006

17. Kontorovich, A. V., \& Nathanson, M. B. (2006). Quadratic addition rules for quantum integers. Journal of Number Theory, 117(1), 1-13. Available at arXiv https://arxiv.org/pdf/math/0503177

18. In a private discussion with the author, Giorgio Kaniadakis pointed out the form (4) of the q-integers as that of the generalized numbers given by Equation 9 of Ref. 8 .

19. Kaniadakis, G. (2001). Non-linear kinetics underlying generalized statistics. Physica A, 296, 405425.

20. Kaniadakis, G. (2002). Statistical mechanics in the context of special relativity. Phys. Rev. E, 66, 056125.

21. Kaniadakis, G. (2005). Statistical mechanics in the context of special relativity II. Phys. Rev. E, 72, 036108.

22. Kaniadakis, G. (2013). Theoretical Foundations and Mathematical Formalism of the Power-Law Tailed Statistical Distributions. Entropy, 15, 3983-4010.

23. Weisstein, Eric W. "Mersenne Number." From MathWorld--A Wolfram Web Resource. http://mathworld.wolfram.com/MersenneNumber.html 